\title{
Fungos conidiais associados ao folhedo de Clusia melchiorii Gleason e C. nemorosa G. Mey. (Clusiaceae) em fragmento de Mata Atlântica, BA, Brasil ${ }^{1}$
}

\author{
Flávia Rodrigues Barbosa ${ }^{2,3,4}$, Leonor Costa Maia² e Luís Fernando Pascholati Gusmão ${ }^{3}$
}

Recebido em 27/08/2007. Aceito em 25/04/2008

\begin{abstract}
RESUMO - (Fungos conidiais associados ao folhedo de Clusia melchiorii Gleason e C. nemorosa G. Mey (Clusiaceae) em fragmento de Mata Atlântica, Bahia, Brasil). Visando ampliar o conhecimento sobre diversidade de fungos conidiais, 10 folhas mortas de três indivíduos de C. melchiorii e de C. nemorosa foram coletadas bimestralmente na Serra da Jibóia, Bahia, no período de outubro/2005 a junho/2006. As folhas foram lavadas em água corrente e mantidas em câmara-úmida durante 30 dias. As estruturas fúngicas foram retiradas para estudo morfológico. Foram identificados 79 táxons de Ascomycota na forma anamórfica. Destes, 78 são hifomicetos e um celomiceto. Do total, $87 \%$ ocorreram sobre C. melchiorii e 55\% sobre C. nemorosa. A maioria dos fungos apresentou freqüência esporádica e constância acidental. As espécies mais freqüentes foram: Beltrania rhombica Penz., Chaetopsina fulva Rambelli, Dactylaria ficusicola Paulus, Gadek \& Hyde, Verticillium theobromae (Turconi) Mason \& Hughes e Volutella sp. 1 (sobre C. melchiorii) e Atroseptaphiale flagelliformis Matsush., Pseudobeltrania sp., Zygosporium gibbum (Sacc., Rousseau \& Bommer) Hughes, Verticillium theobromae (Turconi) Mason \& Hughes e Volutella sp. 1 (sobre C. nemorosa). A similaridade de fungos entre as duas espécies de Clusia atingiu 60\% e 11 táxons foram constantes nos dois hospedeiros: Atrosetaphiale flagelliformis, Beltraniella portoricensis (Stevens) Piroz. \& Patil, Chalara alabamensis Jones \& Ingram., Cryptophiale kakombensis Piroz., Parasympodiella laxa (Subram. \& Vittal), Speiropsis scopiformis Kuthub. \& Nawawi, Thozetella cristata Piroz. \& Hodges, Umbellidion radulans Sutton \& Hodges, Verticillium theobromae, Volutella sp. 2 e Zygosporium gibbum. Os dados mostram que o folhedo produzido por C. melchiorii e C. nemorosa, na Serra da Jibóia, é rico em fungos conidiais. Esses fungos, como decompositores, são importantes para a dinâmica do ecossistema estudado.
\end{abstract}

Palavras-chave: Ascomycota, biodiversidade, freqüência, fungos anamórficos, riqueza

\begin{abstract}
Conidial fungi associated to leaf litter of Clusia melchiorii Gleason and C. nemorosa G. Mey (Clusiaceae) in a fragment of Atlantic rainforest, Bahia State, Brazil). In order to increase the diversity knowledge of conidial fungi, 10 dead leaves from three individuals of C. nemorosa and C. melchiorii were bimonthly collected at the "Serra da Jibóia", State of Bahia, from October/2005 to June/2006. The leaves were washed with tap water and maintained in moist chamber during 30 days. The fungal structures were collected for morphological studies. Seventy nine taxa of Ascomycota, in the anamorphic state were registered: 78 hyphomycete and one Coelomycete. Most of the species occurred on Clusia melchiorii (87\%) and 55\% on C. nemorosa. The majority of the fungi presented sporadical frequency and accidental constancy. The most frequent species were: Beltrania rhombica Penz., Chaetopsina fulva Rambelli, Dactylaria ficusicola Paulus, Gadek \& Hyde, Verticillium theobromae (Turconi) Mason \& Hughes e Volutella sp. 1 (on C. melchiorii) and Atroseptaphiale flagelliformis Matsush., Pseudobeltrania sp., Zygosporium gibbum (Sacc., Rousseau \& Bommer) Hughes, Verticillium theobromae (Turconi) Mason \& Hughes and Volutella sp. 1 (on C. nemorosa). The similarity of fungi between the two species of Clusia reached $60 \%$ and 11 taxa were constant in both hosts: Atrosetaphiale flagelliformis, Beltraniella portoricensis (Stevens) Piroz. \& Patil, Chalara alabamensisJones \& Ingram., Cryptophiale kakombensis Piroz., Parasympodiella laxa (Subram. \& Vittal), Speiropsis scopiformis Kuthub. \& Nawawi, Thozetella cristata Piroz. \& Hodges, Umbellidion radulans Sutton \& Hodges, Verticillium theobromae, Volutella sp. 2 and Zygosporium gibbum. The data show that the litter produced by $C$. melchiorii and C. nemorosa, at the Serra da Jibóia, is rich in conidial fungi. These fungi, as decomposers, are important for the dinamic of the studied ecosystem.
\end{abstract}

Key words: anamorphic, Ascomycota, biodiversity, fungi, frequency, richness

\section{Introdução}

A matéria orgânica acumulada na superfície do solo, constituída por restos de plantas, animais e excrementos funciona como um reservatório de biodiversidade que promove a ciclagem de nutrientes no ecossistema. Dentre os restos vegetais, as folhas constituem a parte mais significativa (Meguro et al. 1979) e os fungos se

\footnotetext{
1 Parte da Dissertação de Mestrado da primeira Autora. Programa de Pós-Graduação em Biologia de Fungos, Universidade Federal de Pernambuco

2 Universidade Federal de Pernambuco, Centro de Ciências Biológicas, Departamento de Micologia. Av. Prof. Nelson Chaves s.n., Cidade Universitária, 50670-420 Recife, PE, Brasil

3 Universidade Estadual de Feira de Santana, Departamento de Ciências Biológicas, Laboratório de Micologia (LAMIC), BR 116, Km 03, 44031-460 Feira de Santana, BA, Brasil

4 Autor para correspondência: faurb10@yahoo.com.br
} 
destacam por contribuir decisivamente para a sua decomposição.

O folhedo tem sido objeto de estudo de micologistas também no Brasil, onde alguns trabalhos se destacam pelo detalhe com que os fungos foram estudados nas seguintes espécies vegetais: Hortia arborea Engl., Licania kunthiana Hook e L. octandra (Hoffmgg. ex R. \& S.) Kuntze (Maia 1983); Cedrela fissilis Vell., Calathea zebrina Lindl., Tibouchina pulchra Cogn. (Grandi \& Gusmão 1995; 1996; 2002; Gusmão \& Grandi 1996; 1997); Alchornea triplinervia (Spreng.) Müll. Arg. e Euterpe edulis Mart. (Grandi 1998; 1999); Miconia cabussu Hoehne (Gusmão et al. 2001) e Caesalpinia echinata Lam. (Grandi \& Silva 2003).

A Serra da Jibóia é um complexo de morros inserido na caatinga, com vegetação constituída por um mosaico de formações vegetacionais que se estende ao longo de seis municípios na região do Recôncavo Sul da Bahia. No topo da serra há presença de Campo Rupestre lato sensu, na porção oriental há predomínio de Floresta Ombrófila Densa e na porção ocidental Floresta Estacional Semi-decidual, Floresta Estacional Decidual e Caatinga Arbórea (M.L.C. Neves 2005, dados não publicados).

Clusia melchiorii Gleason e C. nemorosa G. Mey. constituem um dos componentes principais da vegetação da Serra da Jibóia (Carvalho Sobrinho \& Queiroz 2005). A micota associada a plantas do gênero Clusia foi citada por Farr et al. (1989) que mencionaram, em C. rosea Jacq., seis táxons de fungos. Para as espécies aqui estudadas existem apenas dois registros: Wentiomyces clusiae Bezerra \& Poroca (Ascomycota), em C. melchiorii e Stigmina clusiae M.B. Ellis (anamorfo de Otthia), em C. nemorosa. A primeira é uma espécie fitopatogênica, descrita no Brasil (Mendes et al. 1998), enquanto a segunda foi registrada como decompositora, em Trindade e Tobago (Ellis 1976).

Considerando a importância das espécies de Clusia na área estudada e diante da grande lacuna sobre estudos abordando a micota decompositora, principalmente em áreas de Mata Atlântica, este trabalho teve como objetivo registrar a diversidade de fungos conidiais na Serra da Jibóia fornecendo subsídios para estratégias de preservação ambiental da área.

\section{Material e métodos}

A Serra da Jibóia esta localizada na região do Recôncavo Sul, porção leste do Estado da Bahia, e constitui-se em um complexo de morros. A área de estudo, no Morro da Pioneira, localiza-se no município de Santa Terezinha $\left(12^{\circ} 51^{\prime} \mathrm{S}\right.$ e $\left.35^{\circ} 28^{\prime} \mathrm{W}\right)$ com altitude variando de 700-800 m (M.L.C. Neves 2005, dados não publicados).
Expedições foram realizadas bimestralmente no período de outubro/2005 a junho/2006 para coleta de folhas em decomposição de Clusia melchiorii e C. nemorosa. Durante a primeira expedição foram marcados três indivíduos de cada espécie, tendo como critério a presença de folhedo disponível na base das árvores. Todo o material coletado, nas demais excursões, foi procedente desses mesmos espécimes vegetais. Para a coleta das folhas utilizou-se um quadrado de $50 \mathrm{~cm}^{2} \mathrm{o}$ qual foi arremessado aleatoriamente sobre a serrapilheira e uma folha foi coletada por vez até perfazer 10 folhas por indivíduo de Clusia; essas 10 folhas foram consideradas uma amostra. O material coletado foi submetido à técnica de lavagem em água corrente por uma hora e posteriormente acondicionado em câmarasúmidas sendo estas abertas diariamente, durante 15 minutos, para entrada de ar (R.F. Castañeda-Ruiz 2005, comunicação pessoal). Durante 30 dias as estruturas reprodutivas dos fungos foram coletadas em estereomicroscópio, com auxílio de agulha fina, e colocadas em lâminas permanentes contendo resina PVL (álcool polivinílico + ácido lático + fenol) (Trappe \& Schenck 1982). Exsicatas (lâminas e folhas secas) foram depositadas nos Herbários HUEFS (Universidade Estadual de Feira de Santana) e URM (Departamento de Micologia da Universidade Federal de Pernambuco). Dados de precipitação pluviométrica foram fornecidos pela administração da Reserva Jequitibá, com sede no Município de Elísio Medrado, distando, aproximadamente, $2 \mathrm{~km}$ da área de coleta.

A riqueza foi calculada pelo número de espécies de fungos presentes em cada espécie vegetal. A freqüência de ocorrência foi calculada para os fungos registrados no folhedo de cada espécie de Clusia estudada, de acordo com a fórmula: $\mathrm{F}=\mathrm{n} .100 / \mathrm{N}$ onde, $\mathrm{n}=$ número de amostras em que uma espécie foi registrada; $\mathrm{N}=$ total de amostras em cada espécie de árvore. Foram determinadas as seguintes classes de freqüência: $\mathrm{F} \leq 10 \%=$ Esporádica, $10<\mathrm{F} \leq 30 \%=$ Pouco freqüente, $30<\mathrm{F} \leq 70 \%=$ Freqüente e $\mathrm{F}>70 \%=$ Muito freqüente (Dajoz 1983). Foi considerada uma amostra o conjunto de 10 folhas de cada um dos três indivíduos de cada espécie vegetal, por coleta. A constância foi calculada relacionando o número de coletas em que a espécie foi registrada em cada espécie de Clusia estudada, seguindo a fórmula: $\mathrm{C}=\mathrm{p} .100 / \mathrm{P}$ onde, $\mathrm{p}=$ número de excursões em que um fungo foi encontrado e $\mathrm{P}=$ número total de excursões. Foram definidas as seguintes classes de constância: $\mathrm{C} \leq 25 \%=$ Acidental, $25<\mathrm{C} \leq 50 \%=$ Acessória e $\mathrm{C}>50 \%=$ Constante (Santos \& Cavalcante 1995).

A similaridade da população fúngica entre as duas espécies de Clusia foi estabelecida pelo índice de Sorensen (Krebs 1989): $S=2 c .100 / a+b$ onde, 
$\mathrm{c}=$ número de fungos comuns às duas espécies vegetais, $\mathrm{a}$ e $\mathrm{b}=$ número de fungos presentes sobre $C$. melchiorii e $C$. nemorosa, respectivamente.

\section{Resultados e discussão}

Foram identificados 79 táxons, pertencentes a 47 gêneros de Ascomycota na forma anamórfica. Desses, 78 foram hifomicetos e um celomiceto (Tab. 1).

O predomínio de hifomicetos no folhedo foi observado em pesquisas realizadas no Brasil (Maia 1983; Schoenlein-Crusius \& Milanez 1998; Wellbaum et al. 1999; Gusmão et al. 2001) e em outros países: Porto Rico (Polishook et al. 1996), Austrália (Parungao et al. 2002) e Costa Rica (Bills \& Polishook 1994).

No presente estudo foram encontrados 44 táxons $(55,7 \%)$ associados às folhas de Clusia nemorosa e 69 táxons $(87,3 \%)$ sobre C. melchiorii (Tab. 1).

Além das diferenças entre as espécies vegetais, é provável que a maior riqueza de espécies de fungos encontrada em $C$. melchiorii seja reflexo da maior umidade no local onde o folhedo foi coletado, em comparação à outra área estudada. As plantas de C. melchiorii estão localizadas em área de maior altitude na encosta da Serra, sujeita aos ventos litorâneos, chuvas orográficas e acúmulo de neblina. A vegetação é formada por mata ombrófila densa, que retêm mais umidade, deixando o solo sempre úmido. Os indivíduos de C. nemorosa estão em mata aberta, com clareiras, onde o folhedo e o solo apresentam-se mais secos. Vários fatores, como temperatura, $\mathrm{pH}$, aeração, tempo de senescência, composição química e estrutura das folhas, etc, contribuem, isoladamente ou em conjunto, para o crescimento dos fungos no folhedo (Dix \& Webster 1995). Parungao et al. (2002) também encontraram riqueza diferenciada no material coletado: em uma folha observaram 14 fungos, enquanto em outras nenhum espécime foi encontrado. Para esses autores, a textura das folhas (finas e quebradiças) deve ter influenciado o estabelecimento de fungos.

Menor número de táxons foi observado nas duas primeiras coletas (39 e 34 táxons, respectivamente), sugerindo que o período de estiagem (quatro a seis meses) anterior às coletas, prejudicou o estabelecimento de fungos no folhedo. Nas três coletas seguintes, o número de táxons aumentou (48, 46 e 48 táxons), coincidindo com o início das precipitações. Lodge \& Cantrell (1995) destacam que distúrbios ambientais, como a variação na precipitação, afetam profundamente a distribuição de fungos decompositores em uma área.

A distribuição dos táxons por classe de freqüência evidenciou o predomínio de táxons esporádicos e pouco freqüentes, tanto em folhedo de C. melchiorii (28 e 23 táxons, respectivamente) quanto de C. nemorosa (18 e 12 táxons, respectivamente). Na categoria freqüente foram observados 13 táxons $(18,8 \%)$ sobre C. melchiorii e nove $(20,4 \%)$ sobre $C$. nemorosa. Apenas oito táxons foram muito frequentes nas amostras: Beltrania rhombica, Chaetopsina fulva e Dactylaria ficusicola em C. melchiorii; Atrosetaphiale flagelliformis, Pseudobeltrania sp. e Zygosporium gibbum em C. nemorosa; Verticillium theobromae e Volutella sp. 1 nas duas espécies vegetais.

Beltrania rhombica foi referida em mais de $30 \%$ das amostras de folhedo examinadas por Parungao et al. (2002), na Austrália, enquanto espécies de Verticillium e Volutella foram freqüentes em quatro amostras de folhedo misto em Porto Rico (Polishook et al. 1996).

A maioria das espécies foi classificada como acidental tanto sobre $C$. melchiorii $(43,5 \%)$ quanto sobre C. nemorosa $(45,5 \%)$. Contudo, muitos táxons foram constantes nas duas espécies vegetais: Atrosetaphiale flagelliformis, Beltraniella portoricensis, Chalara alabamensis, Cryptophiale kakombensis, Parasympodiella laxa, Speiropsis scopiformis, Thozetella cristata, Umbellidion radulans, Verticillium theobromae, Volutella sp. 2 e Zygosporium gibbum. Os dados confirmam que certas espécies de fungos conidiais se estabelecem sobre o folhedo em condições específicas (temperatura, umidade, tempo de decomposição do folhedo, etc.), enquanto outras suportam condições mais variadas. Com constância acessória foram registrados 12 táxons sobre folhedo de $C$. melchiorii e seis táxons sobre C. nemorosa.

Os poucos registros da micota sobre Clusia (Farr et al. 1989) não comparam as populações encontradas. A similaridade de espécies de fungos entre $C$. melchiorii e C. nemorosa atingiu $60 \%$. Outros registros de similaridade são referentes a estudos realizados com Guarea guidonea Sleumer e Malnilkara bidentata Chev., onde a similaridade chegou a $32 \%$ e $26 \%$, respectivamente (Polishook et al. 1996) e com populações de fungos presentes em folhas de Licania octandra, L. kunthiana e Hortia arborea. Neste caso, as espécies de fungos presentes em L.octandra e L. kunthiana foram mais semelhantes entre si (70\%) do que com a população em Hortia arborea (64\% e 63,5\%, respectivamente) (Maia 1983). Espécies do mesmo gênero podem ter características que contribuem para a "seleção" de populações de fungos mais similares nos seus respectivos folhedos, porém estudos suplementares são necessários para confirmar essa possibilidade.

Os resultados revelam que o folhedo, produzido por Clusia melchiorii e C. nemorosa na Serra da Jibóia, é rico em fungos conidiais, porém cada táxon, em geral, 
Tabela 1. Fungos conidiais identificados em folhedo de C. melchiorii Gleason e C. nemorosa G. Mey. na Serra da Jibóia, BA, Brasil, no período de outubro/2005 a junho/2006.

\begin{tabular}{|c|c|c|c|c|c|c|c|c|c|c|c|}
\hline \multirow{3}{*}{ Espécies } & \multicolumn{5}{|c|}{ Clusia melchiorii } & \multicolumn{5}{|c|}{ Clusia nemorosa } & \multirow{3}{*}{ Total } \\
\hline & \multicolumn{5}{|c|}{ Coletas } & \multicolumn{5}{|c|}{ Coletas } & \\
\hline & 01 & 02 & 03 & 04 & 05 & 01 & 02 & 03 & 04 & 05 & \\
\hline Anungitea globosa Sutton \& Hodges & & & & & $\mathrm{X}$ & & & & & & 1 \\
\hline Ardhachandra cristaspora (Matsush.) Subram. \& Sudha & $\mathrm{X}$ & $\mathrm{X}$ & $X$ & $\mathrm{X}$ & $\mathrm{X}$ & & & & & & 5 \\
\hline A. selenoides (de Hoog) Subram. \& Sudha & $\mathrm{X}$ & & $\mathrm{X}$ & $\mathrm{X}$ & & & & & & & 3 \\
\hline Atrosetaphiale flagelliformis Matsush. & $\mathrm{X}$ & $\mathrm{X}$ & $\mathrm{X}$ & $\mathrm{X}$ & $\mathrm{X}$ & $\mathrm{X}$ & $\mathrm{X}$ & $\mathrm{X}$ & $\mathrm{X}$ & $\mathrm{X}$ & 10 \\
\hline Beltrania querna Harkn. & $\mathrm{X}$ & & $\mathrm{X}$ & $\mathrm{X}$ & $\mathrm{X}$ & $\mathrm{X}$ & & $\mathrm{X}$ & & & 6 \\
\hline B. rhombica Penz. & $\mathrm{X}$ & $\mathrm{X}$ & $\mathrm{X}$ & $\mathrm{X}$ & $\mathrm{X}$ & & $\mathrm{X}$ & & & & 6 \\
\hline Beltrania sp. 1 & & & $\mathrm{X}$ & & $\mathrm{X}$ & & & & & & 2 \\
\hline Beltrania sp. 2 & & & $\mathrm{X}$ & $\mathrm{X}$ & & & & & & & 2 \\
\hline Beltraniella amoena Castañeda, Cano \& Guarro & & & & $\mathrm{X}$ & $\mathrm{X}$ & & & & & & 2 \\
\hline B. portoricensis (Stevens) Piroz. \& Patil & $\mathrm{X}$ & $\mathrm{X}$ & $\mathrm{X}$ & & $\mathrm{X}$ & $\mathrm{X}$ & $\mathrm{X}$ & $\mathrm{X}$ & $\mathrm{X}$ & $\mathrm{X}$ & 9 \\
\hline Beltraniopsis sp. & $\mathrm{X}$ & & $\mathrm{X}$ & & & & & & & & 2 \\
\hline Chaetendophragmia triangularis Matsush. & & & & $\mathrm{X}$ & & & & & & & 1 \\
\hline Chaetopsina fulva Rambelli & $\mathrm{X}$ & $\mathrm{X}$ & $\mathrm{X}$ & $\mathrm{X}$ & $\mathrm{X}$ & & & & & $\mathrm{X}$ & 6 \\
\hline Chalara alabamensis Jones \& Ingram. & $\mathrm{X}$ & $\mathrm{X}$ & & & $\mathrm{X}$ & $\mathrm{X}$ & $\mathrm{X}$ & $\mathrm{X}$ & & $\mathrm{X}$ & 7 \\
\hline Circinotrichum falcatisporum Piroz. & & & & & & & & & & $\mathrm{X}$ & 1 \\
\hline C. olivaceum (Speg.) Piroz. & & & $\mathrm{X}$ & & & $\mathrm{X}$ & $\mathrm{X}$ & $\mathrm{X}$ & $\mathrm{X}$ & $\mathrm{X}$ & 6 \\
\hline C. papakurae Hughes \& Piroz. & & & $\mathrm{X}$ & $\mathrm{X}$ & & & & & & $\mathrm{X}$ & 3 \\
\hline Cladosporium cladosporioides (Fresen.) de Vries & & & & & $\mathrm{X}$ & & & & & & 1 \\
\hline C. oxysporum Berk. \& Curtis & & & & & & $\mathrm{X}$ & & & & & 1 \\
\hline Clonostachys compactiuscula (Sacc.) Hawksworth \& Gams & & $\mathrm{X}$ & $\mathrm{X}$ & $\mathrm{X}$ & $\mathrm{X}$ & & $\mathrm{X}$ & & & & 5 \\
\hline Cryptophiale guadalcanalensis Matsush. & & & & & $\mathrm{X}$ & & & & & $\mathrm{X}$ & 2 \\
\hline C. kakombensis Piroz. & $\mathrm{X}$ & $\mathrm{X}$ & $\mathrm{X}$ & $\mathrm{X}$ & $\mathrm{X}$ & $\mathrm{X}$ & $\mathrm{X}$ & $\mathrm{X}$ & $\mathrm{X}$ & $\mathrm{X}$ & 10 \\
\hline C. udagawae Piroz. \& Ichinoe & $\mathrm{X}$ & & & & & $\mathrm{X}$ & $\mathrm{X}$ & $\mathrm{X}$ & $\mathrm{X}$ & $\mathrm{X}$ & 6 \\
\hline Cryptophialoidea fasciculata Kuthub. \& Nawawi & & & & & & $\mathrm{X}$ & & $\mathrm{X}$ & & & 2 \\
\hline Curvularia senegalensis (Speg.) Subram. & & & $\mathrm{X}$ & & & & & & & & 1 \\
\hline Cylindrocladium candelabrum Viégas & & & $\mathrm{X}$ & & & & & & & & 1 \\
\hline C. naviculatum Crous \& Wingf. & $\mathrm{X}$ & & & & & & & & & & 1 \\
\hline Dactylaria belliana Paulus, Gadek \& Hyde & & & & & & $\mathrm{X}$ & & & & & 1 \\
\hline D. ficusicola Paulus, Gadek \& Hyde & $\mathrm{X}$ & $\mathrm{X}$ & $\mathrm{X}$ & $\mathrm{X}$ & $\mathrm{X}$ & & & & & & 5 \\
\hline D. fusiformis Shearer \& Crane & & $\mathrm{X}$ & $\mathrm{X}$ & $\mathrm{X}$ & $\mathrm{X}$ & & & & & & 4 \\
\hline Dictyochaeta sp. 1 & & & $\mathrm{X}$ & & & & & & & & 1 \\
\hline Dictyochaeta sp. 2 & & & $\mathrm{X}$ & & & & & & & & 1 \\
\hline Dictyochaeta sp. 3 & & & & $\mathrm{X}$ & & & & & & & 1 \\
\hline Dictyosporium bulbosum Tzean \& Chen & & & & & $\mathrm{X}$ & & & & & & 1 \\
\hline D. digitatum Chen, Hwang \& Tzean & & & & $\mathrm{X}$ & & & & & & & 1 \\
\hline D. elegans Corda & & $\mathrm{X}$ & $\mathrm{X}$ & $\mathrm{X}$ & & & & & & $\mathrm{X}$ & 4 \\
\hline Diplocladiella cornitumida Barbosa, Gusmão \& Castañeda & & & & $\mathrm{X}$ & & & & & & & 1 \\
\hline Flosculomyces floridaensis Sutton & & & & & & & $\mathrm{X}$ & $\mathrm{X}$ & $\mathrm{X}$ & $\mathrm{X}$ & 4 \\
\hline Guedea novae-zelandiae Hughes & & & & & & & & & & $\mathrm{X}$ & 1 \\
\hline Gyrothrix circinata (Berk. \& Curtis) Hughes & & & & & & & $\mathrm{X}$ & $\mathrm{X}$ & & & 2 \\
\hline G. magica Lunghini \& Onofri & & $\mathrm{X}$ & $\mathrm{X}$ & $\mathrm{X}$ & $\mathrm{X}$ & & & & & & 4 \\
\hline G. microsperma (Höhn.) Piroz. & & & $\mathrm{X}$ & $\mathrm{X}$ & & & & & & $\mathrm{X}$ & 3 \\
\hline G. verticiclada (Goid.)Hughes \& Piroz. & & $\mathrm{X}$ & & $\mathrm{X}$ & $\mathrm{X}$ & & & $\mathrm{X}$ & & $\mathrm{X}$ & 5 \\
\hline Gyrothrix sp. 1 & & & & & $\mathrm{X}$ & & & & & & 1 \\
\hline Henicospora coronata Sutton \& Kirk & $\mathrm{X}$ & & & & & & & & & & 1 \\
\hline H. minor Kirk \& Sutton & & & & & & $\mathrm{X}$ & & & & & 1 \\
\hline Idriella ramosa Matsush. & & & $\mathrm{X}$ & & & $\mathrm{X}$ & & & & $\mathrm{X}$ & 3 \\
\hline I. setiformis Castañeda \& Arnold & & & $\mathrm{X}$ & $\mathrm{X}$ & & $\mathrm{X}$ & $\mathrm{X}$ & $\mathrm{X}$ & & $\mathrm{X}$ & 6 \\
\hline Kylindria pluriseptata Castañeda & $\mathrm{X}$ & $\mathrm{X}$ & & $\mathrm{X}$ & & & & & & & 3 \\
\hline Kionochaeta ramifera (Matsush.) Kirk \& Sutton & $\mathrm{X}$ & $\mathrm{X}$ & $\mathrm{X}$ & & & & & & & & 3 \\
\hline Paliphora intermedia Alcorn & $\mathrm{X}$ & & & & & & & & & & 1 \\
\hline Paraceratocladium polysetosum Castañeda & $\mathrm{X}$ & & & & & $\mathrm{X}$ & & & & & 2 \\
\hline P. silvestre Castañeda & & & $\mathrm{X}$ & $\mathrm{X}$ & & & & & & & 2 \\
\hline Parasympodiella laxa (Subram. \& Vittal) Ponnappa & & $\mathrm{X}$ & $\mathrm{X}$ & $\mathrm{X}$ & $\mathrm{X}$ & & $\mathrm{X}$ & $\mathrm{X}$ & & $\mathrm{X}$ & 7 \\
\hline Pleurophragmium varieseptatum Matsush. & & & & & & & & $\mathrm{X}$ & & & 1 \\
\hline Pseudobeltrania sp. & $\mathrm{X}$ & & & & & $\mathrm{X}$ & $\mathrm{X}$ & $\mathrm{X}$ & $\mathrm{X}$ & $\mathrm{X}$ & 6 \\
\hline
\end{tabular}


Tabela 1 (continuação)

\begin{tabular}{|c|c|c|c|c|c|c|c|c|c|c|c|}
\hline \multirow{3}{*}{ Espécies } & \multicolumn{5}{|c|}{ Clusia melchiorii } & \multicolumn{5}{|c|}{ Clusia nemorosa } & \multirow{3}{*}{ Tota } \\
\hline & \multicolumn{5}{|c|}{ Coletas } & \multicolumn{5}{|c|}{ Coletas } & \\
\hline & 01 & 02 & 03 & 04 & 05 & 01 & 02 & 03 & 04 & 05 & \\
\hline Pseudobotrytis terrestris (Timonin) Subramanian & & $\mathrm{X}$ & & & & & $\mathrm{X}$ & & & & 2 \\
\hline Satchmopsis brasiliensis Sutton \& Hodges & & & & $\mathrm{X}$ & $\mathrm{X}$ & & & & & $\mathrm{X}$ & 3 \\
\hline Selenodriella fertilis (Piroz. \& Hodges) Castañeda \& Kendr. & & & & $\mathrm{X}$ & & & & & & & 1 \\
\hline S. perramosa Kendr. \& Castañeda & & $\mathrm{X}$ & $\mathrm{X}$ & $\mathrm{X}$ & $\mathrm{X}$ & $\mathrm{X}$ & & & $\mathrm{X}$ & & 6 \\
\hline Selenosporella curvispora Arnaud & & $\mathrm{X}$ & & & & $\mathrm{X}$ & & & & & 2 \\
\hline Speiropsis scopiformis Kuthub. \& Nawawi & $\mathrm{X}$ & $\mathrm{X}$ & $X$ & $X$ & $\mathrm{X}$ & $\mathrm{X}$ & $\mathrm{X}$ & & & $\mathrm{X}$ & 8 \\
\hline Sporendocladia bactrospora (Kendrick) Wingf. & & & & & $\mathrm{X}$ & $\mathrm{X}$ & & & $\mathrm{X}$ & $\mathrm{X}$ & 4 \\
\hline Stachybotrys kampalensis Hansf. & & & $\mathrm{X}$ & & & & & & & & 1 \\
\hline S. longispora Matsush. & & & $\mathrm{X}$ & $\mathrm{X}$ & $\mathrm{X}$ & & & & & & 3 \\
\hline S. parvispora Hughes & & $\mathrm{X}$ & & & & $\mathrm{X}$ & & & & & 2 \\
\hline Subulispora longirostrata Nawawi \& Kuthub. & & & $X$ & & & & & & & & 1 \\
\hline Thozetella cristata Piroz. \& Hodges & $\mathrm{X}$ & $\mathrm{X}$ & $\mathrm{X}$ & $\mathrm{X}$ & $\mathrm{X}$ & $\mathrm{X}$ & $\mathrm{X}$ & & & $\mathrm{X}$ & 8 \\
\hline T. cubensis Castañeda \& Arnold & & & & $\mathrm{X}$ & & & & & & & 1 \\
\hline Umbellidion radulans Sutton \& Hodges & & $\mathrm{X}$ & & $\mathrm{X}$ & $\mathrm{X}$ & $\mathrm{X}$ & $\mathrm{X}$ & & & $\mathrm{X}$ & 6 \\
\hline $\begin{array}{l}\text { Vermiculariopsiella cornuta (Rao \& de Hoog) Nawawi, } \\
\text { Kuthub. \& Sutton }\end{array}$ & $\mathrm{X}$ & & $\mathrm{X}$ & & $\mathrm{X}$ & & & & & & 3 \\
\hline Verticillium theobromae (Turconi) Mason \& Hughes & $\mathrm{X}$ & $\mathrm{X}$ & $\mathrm{X}$ & $\mathrm{X}$ & $\mathrm{X}$ & $\mathrm{X}$ & $\mathrm{X}$ & $\mathrm{X}$ & $\mathrm{X}$ & $\mathrm{X}$ & 10 \\
\hline Volutella minima Höhn. & & & & $\mathrm{X}$ & $\mathrm{X}$ & $\mathrm{X}$ & & & & & 3 \\
\hline Volutella sp. 1 & $\mathrm{X}$ & $\mathrm{X}$ & $\mathrm{X}$ & $\mathrm{X}$ & $\mathrm{X}$ & $\mathrm{X}$ & $\mathrm{X}$ & $\mathrm{X}$ & $\mathrm{X}$ & $\mathrm{X}$ & 10 \\
\hline Volutella sp. 2 & & & & $X$ & $\mathrm{X}$ & & & & & & 2 \\
\hline Wiesneriomyces laurinus (Tassi) Kirk & & & & & $\mathrm{X}$ & & & & & & 1 \\
\hline Zygosporium echinosporum Bunting \& Mason & & & & & & $\mathrm{X}$ & $\mathrm{X}$ & $\mathrm{X}$ & $\mathrm{X}$ & $\mathrm{X}$ & 5 \\
\hline Z. gibbum (Sacc., Rousseau \& Bommer) Hughes & & $\mathrm{X}$ & $\mathrm{X}$ & $\mathrm{X}$ & $X$ & $\mathrm{X}$ & $X$ & $\mathrm{X}$ & $\mathrm{X}$ & $\mathrm{X}$ & 9 \\
\hline Z. masonii Hughes & & & $\mathrm{X}$ & $\mathrm{X}$ & $\mathrm{X}$ & & & $\mathrm{X}$ & & & 4 \\
\hline Total & 24 & 26 & 39 & 39 & 36 & 27 & 21 & 20 & 13 & 28 & 273 \\
\hline
\end{tabular}

tem baixa freqüência e constância no substrato. Esses dados preliminares e pioneiros devem subsidiar estudos de natureza mais ampla, contribuindo para melhor entendimento da importância dos fungos conidiais na decomposição do folhedo.

\section{Agradecimentos}

À Coordenação de Aperfeiçoamento do Pessoal de Nível Superior (CAPES), pela bolsa de Mestrado (Programa de Pós-Graduação em Biologia de Fungos/UFPE), concedida ao primeiro autor; ao Conselho Nacional de Desenvolvimento Científico e Tecnológico (CNPq), pelos auxílios concedidos ao segundo (Bolsa PQ) e terceiro autores, no Edital Universal (proc. 471619/04-3).

\section{Referências bibliográficas}

Bills, G.F. \& Polishook, J.D. 1994. Abundance and diversity of microfungi in leaf litter of a lowland rainforest in Costa Rica. Mycologia 86: 187-198.
Carvalho Sobrinho, J.G. \& Queiroz, L.P. 2005. Composição florística de um fragmento de Mata Atlântica na Serra da Jibóia, Santa Terezinha, Bahia Brasil. Sitientibus 5: 20-27.

Dajoz, R. 1983. Ecologia Geral. Rio de Janeiro, Ed. Vozes.

Dix, N.I. \& Webster, J. 1995. Fungal Ecology. Cambridge, University Press.

Ellis, M.B. 1976. More Dematiaceous Hyphomycetes. Kew, Commonwealth Mycological Institute.

Farr, D.F.; Bills, G.F.; Chamuris, G.P. \& Rossman, A.Y. 1989. Fungi on Plants and Plant Products in the United States. St. Paul, The American Phytopatological Society, APS Press.

Grandi, R.A.P. 1998. Hyphomycetes decompositores do folhedo de Alchornea triplinervea (Spreng.) Mull. Arg. Hoehnea 25: 133-148.

Grandi, R.A.P. 1999. Hifomicetes decompositores do folhedo de Euterpe edulis Mart. Hoehnea 26: 87-101.

Grandi, R.A.P. \& Gusmão, L.F.P. 1995. Espécies de Gyrothrix (Hyphomycetes) no folhedo de Cedrela fissilis Vell. em Maringá, PR, Brasil. Hoehnea 22: 191-196.

Grandi, R.A.P. \& Gusmão, L.F.P. 1996. Hyphomycetes decompositores de raízes de Calathea zebrina (Sims) Lindl. (Matantaceae), provenientes da Reserva Biológica do Alto da Serra de Paranapiacava, Santo André, SP, Brasil. Revista Brasileira de Botânica 19: 165-172. 
Grandi, R.A.P. \& Gusmão, L.F.P. 2002. Hyphomycetes decompositores do folhedo de Tibouchina pulchra Cogn. Revista Brasileira de Botânica 25: 79-87.

Grandi, R.A.P. \& Silva, T.V. 2003. Hyphomycetes sobre folhas de Caesalpinia echinata Lam.: ocorrências novas para o Brasil. Revista Brasileira de Botânica 26: 489-493.

Gusmão, L.F.P. \& Grandi, R.A.P. 1996. Espécies do grupo Beltrania (Hyphomycetes) associadas à folhas de Cedrela fissilis Vell. (Meliaceae) em Maringá, PR, Brasil. Hoehnea 23: 91-102.

Gusmão, L.F.P. \& Grandi, R.A.P. 1997. Hyphomycetes com conidioma dos tipos esporodóquio e sinema associados à folhas de Cedrela fissilis (Meliaceae), em Maringá, PR, Brasil. Revista Brasileira de Botânica 11: 123-133.

Gusmão, L.F.P.; Grandi, R.A.P. \& Milanez, A.I. 2001. Hyphomycetes from leaf litter of Miconia cabussu in the Brazilian Atlantic rain forest. Mycotaxon 79: 201-213.

Krebs, C.J. 1989. Ecological Methodology. New York, Harper \& Row Publishers.

Lodge, D.J. \& Cantrell, S. 1995. Fungal communities in wet tropical forests: variation on time and space. Canadian Journal of Botany 73: 1391-1398.

Maia, L.C. 1983. Sucessão de fungos em folhedo de Floresta Tropical Úmida. Recife, Ed. Universitária, UFPE.

Meguro, M.; Vinueza, G.N. \& Delitti, W.B.C. 1979. Ciclagem de nutrientes minerais na mata mesófila secundária - São Paulo. I - Produção e conteúdo de nutrientes minerais no folhedo. Boletim de Botânica da Universidade de São Paulo 7: 11-31.
Mendes, M.A.S.; Silva, V.L.; Dianese, J.C.; Ferreira, M.A.S.V.; Santos, C.E.N.; Gomes Neto, E.; Urben, A.F. \& Castro, C. 1998. Fungos em Plantas no Brasil. Brasília, Embrapa-SPI/EmbrapaCenargen.

Parungao, M.M.; Fryar, S.C. \& Hyde K.D. 2002. Diversity of fungi on rainforest litter in North Queensland, Austrália. Biodiversity and Conservation 11: 1185-1194.

Polishook, J.D.; Bills, G.F. \& Lodge, D.J. 1996. Microfungi from decaying leaves of two rain forest trees in Puerto Rico. Journal of Industrial Microbiology 17: 284-294.

Santos, E.J. \& Cavalcanti, L.H. 1995. Myxomycetes ocorrentes em bagaço de cana armazenado em indústria. Revista Brasileira de Plantas Medicinais 67: 05-22.

Schoenlein-Crusius, I.H. \& Milanez, A.I. 1998. Fungal succession on leaves of Alchornea triplinervia (Spreng.) Muell. Arg. submerged in a stream of an Atlantic Rainforest in the State of São Paulo, Brazil. Revista Brasileira de Botânica 21: 253-259.

Trappe, J.M. \& Schenck, N.C. 1982. Taxonomy of the fungi forming Endomycorrhizae. Pp. 1-9. In: N.C. Schenck (ed.). Methods and Principles of Mycorrhizae Research. St Paul, The American Phytopatological Society.

Wellbaum, L.; Schoenlein-Crusius I.H. \& Santos, V.B. 1999. Fungos filamentosos em folhas de ambiente terrestre e aquático da Ilha dos Eucaliptos, Represa do Guarapiranga, São Paulo, SP. Revista Brasileira de Botânica 22: 69-74 